\title{
UNILATERAL VARIATION OF PLANTARIS MUSCLE - A CASE REPORT
}

Phalguni Srimani ${ }^{1}$, Rudradev Meyur ${ }^{2}$, Alpana De (Bose) ${ }^{3}$, Banani Kundu ${ }^{4}$, Anirban Sadhu ${ }^{5}$

\section{HOW TO CITE THIS ARTICLE:}

Phalguni Srimani, Rudradev Meyur, Alpana De (Bose), Banani Kundu, Anirban Sadhu. "Unilateral Variation of Plantaris Muscle - A Case Report". Journal of Evolution of Medical and Dental Sciences 2014; Vol. 3, Issue 03, January 20; Page: 618-622, DOI: 10.14260/JEMDS/2014/1875

ABSTRACT: Plantaris is considered as vestigial muscle in human with a small belly and long thin tendon. It is known to present several anatomical variations in terms of its occurrence, origin, course, relation with surrounding neurovascular structures and insertion. It may be absent unilaterally or bilaterally. On rare occasion, its double occurrence has been reported also. One such variation was observed unilaterally in the present report in which variant origin with two distinct heads of the muscle were seen to be merged to form a common long tendon that passed deep to both heads of gastrocnemius and soleus before final insertion into flexor retinaculum of ankle. Despite its vestigial nature, injury to plantaris muscle can present diagnostic challenge among clinicians and radiologists. Surgical importance of the muscle lies in successful use of its tendon for reconstructive surgery. Therefore, knowledge of possible variations regarding plantaris muscle is important.

KEY WORDS: Plantaris, Vestigial, Additional head, Flexor retinaculum.

INTRODUCTION: Plantaris belongs to superficial group of flexor muscles of posterior crural compartment along with gastrocnemius and soleus and thus form the bulk of the calf. The muscle takes origin from lower part of lateral supracondylar line and oblique popliteal ligament. It becomes tendinous approximately at the level of origin of soleus from tibia in the proximal part of lower leg and the long thin tendon then passes downwards between medial head of gastrocnemius and soleus and is further continued along medial aspect of Achilles tendon. Finally, it gets inserted either independently or in association with Achilles tendon into calcaneus. Due to structural resemblance of its slender tendon with nerve, it is often described as 'freshman's nerve'. It is regarded as weak flexor of knee and acts with gastrocnemius and soleus, thereby assisting in plantar flexion of ankle also. Due to presence of high density muscle spindles, it carries proprioceptive function for larger more powerful plantar flexors. Clinically, both the muscle belly and its tendon can be palpated respectively in popliteal fossa and along medial aspect of Achilles tendon near its insertion. It has been reported that nerve to plantaris arises from tibial nerve as independent branch or via nerve to lateral head of gastrocnemius ${ }^{1,2}$.

Plantaris muscle has been observed to present frequent variations in terms of its occurrence, origin, course, relation with surrounding neurovascular structures and insertion. MRI study was compared to cadaveric study to evaluate the incidence of the muscle 3. Prevalence of accessory muscle was determined by MRI as $6.3 \%$ with no significant difference between sexes in which origin of all accessory muscles were found to be merged with normal plantaris except one which got merged with lateral head of gastrocnemius ${ }^{4}$. The muscle was found to be present in all 20 legs but having different morphology of insertion having dorsal and ventral belly of the muscle ${ }^{5}$. The muscle may be absent in $10 \%$ cases $^{1,6}$. On rare occasions, its unilateral 7,8 and bilateral double presence 9,10 have been described. Therefore, knowledge of possible variations regarding plantaris muscle is important. 
The aim of our study is to present a report of unilateral variation of plantaris muscle in right lower limb.

CASE REPORT: During routine dissection of right lower limb involving popliteal region and posterior crural region of an approximately 65year-old male cadaver for $1^{\text {st }}$ year MBBS student showed the following unusual findings:

Plantaris muscle was observed to be arising by two heads. One head (lateral) was thicker and arising from lower part of lateral supra condylar line deep to lateral head of gastrocnemius and the other head (medial) was comparatively smaller and originated from lower and medial aspect of oblique popliteal ligament deep to medial head of gastrocnemius (Figure 1). Very few fibers from lateral head were seen to have connected with lateral head of gastrocnemius. After origin, lateral head was directed downwards and medially and found to join soon with medial head which was directed downwards and laterally to form a common slender tendon. The tendon was seen passing downwards deep to both heads of gastrocnemius and soleus (Figure 1). The tendon was further followed throughout its course to locate its distal attachment. Finally, it was seen along medial border of tendocalcaneus to be inserted into the flexor retinaculum of ankle (Figure 2). Meticulous dissection on the left side revealed no such variation regarding plantaris muscle.

DISCUSSION: Plantaris muscle is known as vestigial muscle in human as its distal attachment has shifted secondarily well short of plantar aponeurosis to calcaneus due to process of evolution for erect posture and bipedal locomotion ${ }^{1}$. Embryologically, it is considered as a derivative of deeper portion of lateral head of gastrocnemius and often represented as third head of gastrocnemius or 'gastrocnemius tertius'. Our observation regarding connection of few fibers of lateral head of this muscle with lateral head of gastrocnemius thus could support the embryological explanations advocated by McMurrich ${ }^{11}$.

Anatomical variations of the muscle are not uncommon. Lower part of lateral supracondylar line and posterior surface of lateral condyle of femur have been considered bilaterally as bicipital origin of the muscle. Tendon of such muscle was seen to be merged with calcanean tendon ${ }^{10}$. A distinct anomalous muscle on medial side of right leg was documented as second plantaris due to its morphological resemblance ${ }^{8}$. Sawant SP et al 7 has described a rare variation of the muscle with presence of two heads taking origin from lower part of lateral supracondylar line and oblique popliteal ligament similar to our observations. The muscle with bilateral existence of two separate bellies was reported by Rana et al ${ }^{9}$. In all previous reports, plantaris tendon was observed to pass between gastrocnemius and soleus. However, we observed a rare course of common tendon passing deep to both heads of gastrocnemius and soleus. Surgical intervention without knowledge of such deep seated plantaris tendon may lead to inadvertent damage to surrounding structures. Multiple variable insertions along with some rare variety including iliotibial band, lateral patellar retinaculum, iliotibial tract or as split attachments on posteromedial side of calcaneus have been described ${ }^{4,8}$. Other than its usual bony insertion to calcaneus, additional muscle with its tendon may be inserted into crural fascia also ${ }^{9}$. In this regard, we followed the common tendon to be fused finally with flexor retinaculum which was also found in $28.84 \%$ cases in a study done previously in adult Indians ${ }^{2}$. 
Despite of its vestigial nature, documentation of anatomical variation of plantaris muscle is clinically important. Variations in terms of its distinct interdigitations with lateral head of gastrocnemius or having a strong fibrous extension to patella may be responsible for patellofemoral pain syndrome ${ }^{13}$. The muscle may get injured during surgical procedures because of its superficial attachment with fascia of leg and its long tendon resembling to nerve ${ }^{9}$. Its tendon may also get entrapped between tibial nerve and its branch to soleus and produce compression neuropathy ${ }^{14}$. Injury to plantaris muscle and its tendon or associated tears of gastrocnemius, soleus and anterior cruciate ligament may be regarded as important cause of 'Tennis leg'. Rupture of its tendon may be presented as non-specific lower leg pain. Recently, MRI study has focused an isolated complete rupture of the muscle ${ }^{2}$. Correct interpretation of MRI studies for diagnosing such type of clinical presentation needs detailed anatomical knowledge about the muscle not only to identify tense mass between gastrocnemius and soleus as sign of rupture of tendon but also to rule out more severe condition, such as deep vein thrombosis ${ }^{7}$. Awareness regarding variation of the muscle is also important to surgeons undertaking reconstructive surgery. Tendon of plantaris due to its excellent tensile strength has been successfully used as graft for reconstruction of flexor tendon in hand and anterior talofibular and calcaneofibular ligament of ankle 10. It has also been tried for atrioventricular valve repair ${ }^{15}$.

CONCLUSION: Considering the above discussion, though minor differences have been observed between present study and earlier reports related to plantaris, prior knowledge about such possible variation will definitely supplement the understanding of muscular variation of posterior crural region.

ACKNOWLEDGEMENT: Authors sincerely acknowledge faculties of the department of Anatomy for their hands of help.

\section{REFERENCES:}

1. Mahadevan V. Leg. In: Standring S, ed. Gray's Anatomy. The Anatomical Basis of Clinical Practice. 40th Ed., Churchill Livingstone. 2008: 1421.

2. Spine AA. The plantaris muscle: anatomy, injury, imaging and treatment. J Can Chiropr Assoc. 2007; 51: 158-165.

3. Saxena A, Bareither D. Magnetic resonance and cadaveric findings of the incidence of plantaris tendon. Foot Ankle Int. 2000 July; 21(7): 570-572.

4. Herzog RJ. Accessory Plantaris Muscle: Anatomy and Prevalence. HSS J. 2011; 7:52-56.

5. Aragao JA, Reis FP, Guerra DR, Cabral RH. The occurrence of plantaris muscle and its muscletendon relationship in adult human cadavers. Int J Morphol. 2010; 28(1): 255-258.

6. Sharma S, Sharma GD, Bhardwaj S. Absence of plantaris muscle. Novel Science International Journal of Medical Science. 2012; 1(11-12): 300-304.

7. Sawant SP, Shaikh ST, More RM. A rare variation of plantaris muscle. Int J Biol Med Res. 2012; 3(4): 2437-2440.

8. Kwinter DM, Lagrew JP, Kretzer J, Lawrence C, Malik D, Mater M, Brueckner JK. Unilateral double plantaris muscle: A rare anatomical variation. Int J Morphol. 2010; 28(4): 1097-1099. 
9. Rana KK, Das S, Verma R. Double plantaris muscle: A cadaveric study with clinical importance. Int J Morphol. 2006, 24 (3): 495-498.

10. Upasna, Kumar A. Bicipital origin of plantaris muscle - a case report. Int J Anat Var. 2011; 4: 177-179.

11. McMurrich JP. The phylogeny of the crural flexors. Am J Anat. 1905; 4: 33-76.

12. Nayak SR, Krishnamurthy A, Ramanathan L, Ranade AV, Prabhu LV, Jiji PJ, Rai R, Chettiar GK, Potu BK. Anatomy of plantaris muscle: a study in adult Indians. Clin Ter. 2010; 161(3):249252.

13. Freeman AJ, Jacobson NA, Fogg QA. Anatomical variations of the plantaris muscle and a potential role in patellofemoral pain syndrome. Clin Anat. 2008 Mar; 21(2): 178-181.

14. Das S, Basudeva N. Entrapment of plantaris tendon between the tibial nerve and its branch: a case report. Eur J Anat. 2006; 10(3): 53-55.

15. Shauhaiber JH, Shauhaiber HH. Plantaris tendon graft for atrioventricular valve repair. A novel hypothetical technique. Tex

16. Heart Inst J. 2003; 30: 42-44.

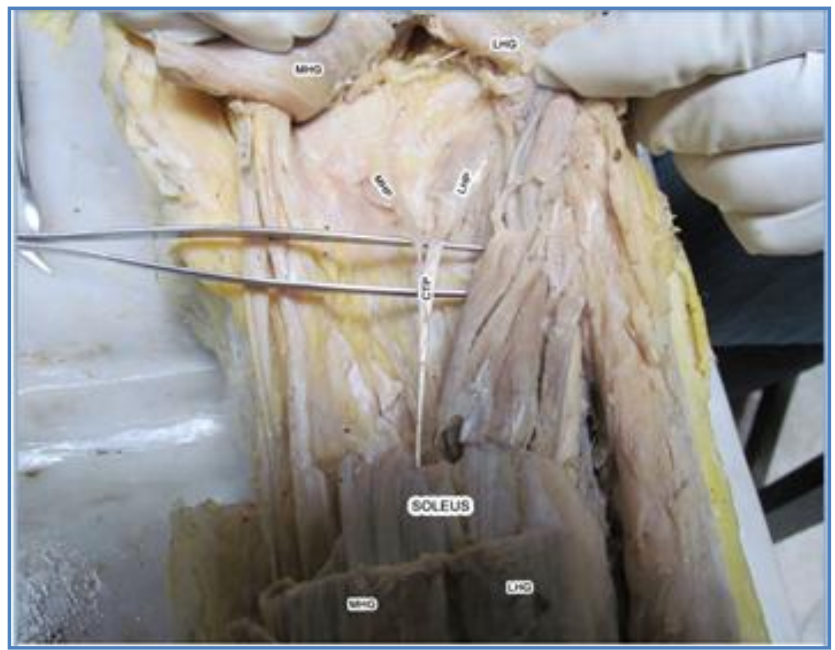

Fig. 1

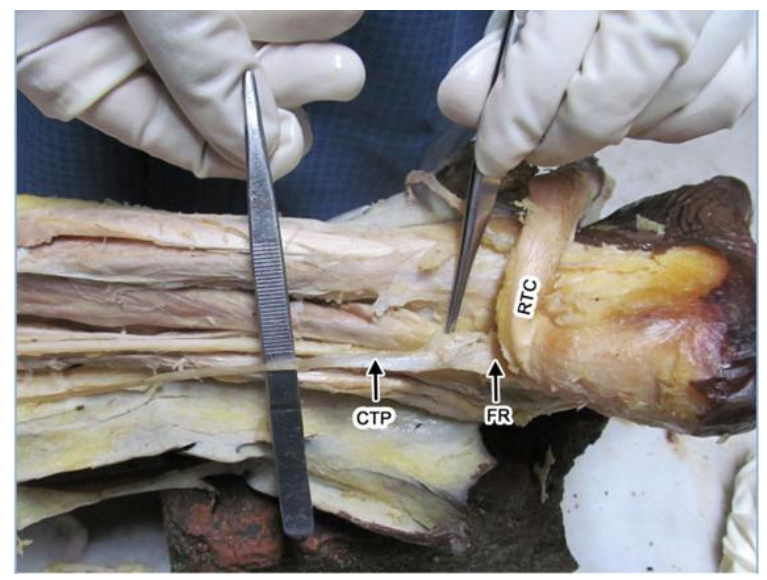

Fig. 2
Lateral head of plantaris (LHP) originating from lower part of lateral supracondylar line and medial head of plantaris (MHP) from oblique popliteal ligament. Common tendon of plantaris (CTP) passing deep to both the heads of gastrocnemius and soleus. (LHG: Lateral head of gastrocnemius; MHG: Medial head of gastrocnemius).

Distal part of common tendon of plantaris (CTP) inserting into flexor retinaculum (FR) of ankle deep to tendocalcaneus. (RTC: Reflected tendocalcaneus). 


\section{AUTHORS:}

1. Phalguni Srimani

2. Rudradev Meyur

3. Alpana De (Bose)

4. Banani Kundu

5. Anirban Sadhu

\section{PARTICULARS OF CONTRIBUTORS:}

1. Post Graduate Student, Department of Anatomy, R.G. Kar Medical College and Hospital, 1, Kshudiram Bose Sarani, Kolkata, West Bengal, India.

2. Associate Professor, Department of Anatomy, R.G. Kar Medical College and Hospital, 1, Kshudiram Bose Sarani, Kolkata, West Bengal, India.

3. Professor and Head, Department of Anatomy, R.G. Kar Medical College and Hospital, 1, Kshudiram Bose Sarani, Kolkata, West Bengal, India.
4. Assistant Professor, Department of Anatomy, R.G. Kar Medical College and Hospital, 1, Kshudiram Bose Sarani, Kolkata, West Bengal, India.

5. Assistant Professor, Department of Anatomy, R.G. Kar Medical College and Hospital, 1, Kshudiram Bose Sarani, Kolkata, West Bengal, India.

\section{NAME ADDRESS EMAIL ID OF THE CORRESPONDING AUTHOR:}

Dr. Phalguni Srimani,

Geetanjali Apartment, Flat No. 101;37A, G.T. Road,

P.O. Rishra, Dist. Hooghly,

PIN - 712248, West Bengal, India.

Email - falgunisreemani@yahoo.co.in

Date of Submission: 18/12/2013.

Date of Peer Review: 20/12/2013.

Date of Acceptance: 07/01/2014.

Date of Publishing: 16/01/2014. 